\title{
X-rays from magnetic massive OB stars
}

\section{Petit ${ }^{1}$, D. H. Cohen ${ }^{2}$, Y. Nazé ${ }^{3}$, M. Gagné ${ }^{4}$, R. H. D. Townsend ${ }^{5}$, M. A. Leutenegger ${ }^{6}$, A. ud-Doula ${ }^{7}$, S. P. Owocki ${ }^{1}$ and G. A. Wade ${ }^{8}$}

${ }^{1}$ Dept. of Physics \& Astronomy, University of Delaware, Newark, DE, USA email: vpetit@udel.edu

${ }^{2}$ Dept. of Physics \& Astronomy, Swarthmore College, Swarthmore, PA, USA

${ }^{3}$ GAPHE Dépt. AGO, Université de Liège, Liège, Belgium

${ }^{4}$ Dept. of Geology \& Astronomy, West Chester University, West Chester, PA, USA

${ }^{5}$ Dept. of Astronomy, University of Wisconsin-Madison, Madison, WI, USA

${ }^{6}$ Laboratory for High Energy Astrophysics, NASA/GSFC, Greenbelt, MD, USA

${ }^{7}$ Penn State Worthington Scranton, Dunmore, PA, USA

${ }^{8}$ Dept. of Physics, Royal Military College of Canada, Kingston, ON, Canada

\begin{abstract}
The magnetic activity of solar-type and low-mass stars is a well known source of coronal X-ray emission. At the other end of the main sequence, X-rays emission is instead associated with the powerful, radiatively driven winds of massive stars. Indeed, the intrinsically unstable line-driving mechanism of OB star winds gives rise to shock-heated, soft emission $(\sim 0.5 \mathrm{keV})$ distributed throughout the wind. Recently, the latest generation of spectropolarimetric instrumentation has uncovered a population of massive OB-stars hosting strong, organized magnetic fields. The magnetic characteristics of these stars are similar to the apparently fossil magnetic fields of the chemically peculiar ApBp stars. Magnetic channeling of these OB stars' strong winds leads to the formation of large-scale shock-heated magnetospheres, which can modify UV resonance lines, create complex distributions of cooled Halpha emitting material, and radiate hard $(\sim 2-5 \mathrm{keV}) \mathrm{X}$-rays. This presentation summarizes our coordinated observational and modelling efforts to characterize the manifestation of these magnetospheres in the X-ray domain, providing an important contrast between the emission originating in shocks associated with the large-scale fossil fields of massive stars, and the X-rays associated with the activity of complex, dynamo-generated fields in lower-mass stars.
\end{abstract}

Keywords. Stars - early-type, Stars - magnetic fields

\section{Introduction}

The connection between the coronal X-ray emission of Sun-like, low-mass stars and their magnetic activity is well established by the strong correlation between their X-ray luminosity and the size of their convection zone, as well as their rotation rates (e.g. Wright et al. 2011). These parameters are the main ingredients powering their magnetic dynamos. Most massive OB stars are also X-ray bright (Berghoefer et al. 1997), although this emission is not traditionally associated with magnetism for two principal reasons.

First, the internal structure of main sequence stars undergoes major changes with increasing mass, transitioning from a radiative core and convective envelope to a convective core and radiative envelope. The best studied population of magnetic stars massive enough to have radiative envelopes are the so-called chemically peculiar ApBp stars. In contrast to the low-mass cool stars, the ApBp stars have strong, large scale, mostly dipolar magnetic fields, and represent only a sub-population $(\sim 10 \%)$ of all the A-type and late-B type stars (Power 2007). Recent efforts to characterize the magnetic properties of 

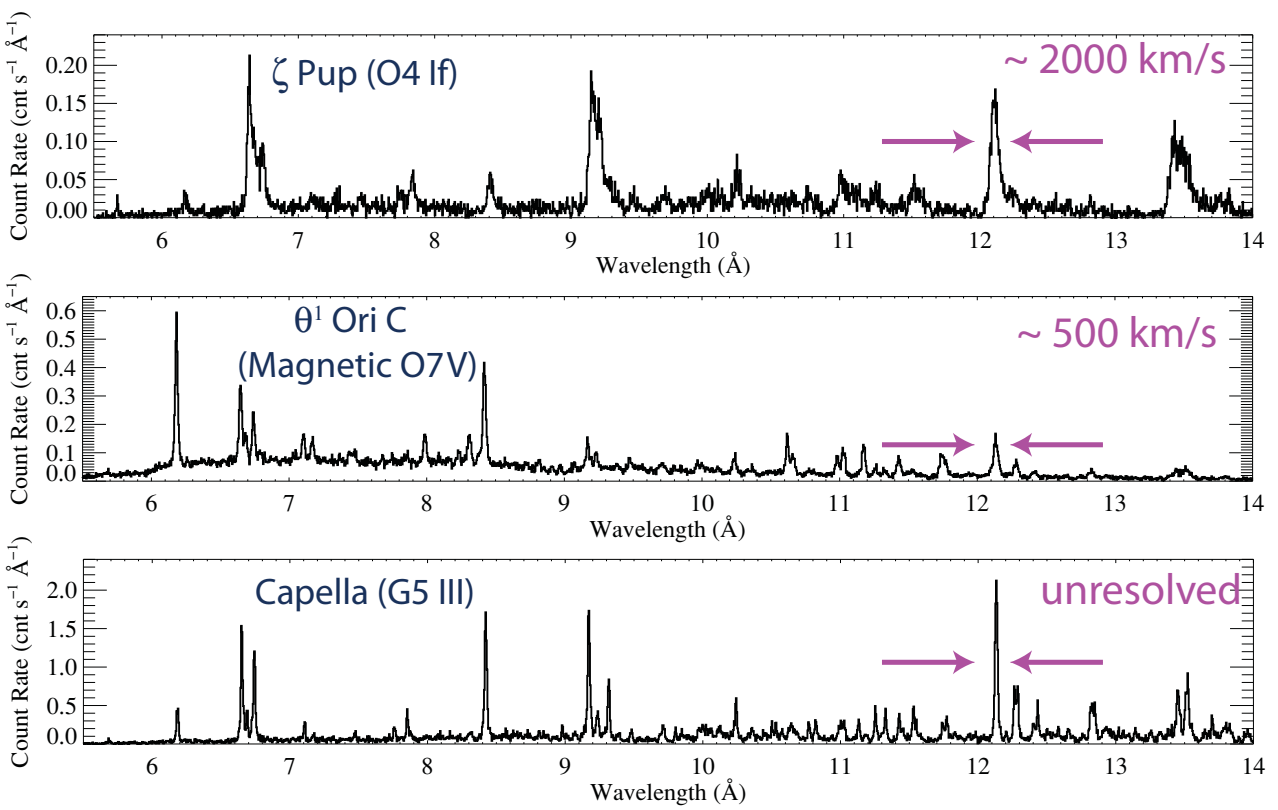

Figure 1. Chandra X-ray observatory High Energy Transmission Grating (HETG) spectra of the non-magnetic O-type type star $\zeta$ Pup (top), the magnetic O-type star $\theta^{1}$ Ori C (middle), and the coronal emission from the G-type star Capella (bottom).

more massive OB stars, such as the large survey performed by the Magnetism in Massive Stars Project (Wade et al. 2011), have shown that all OBA-type stars share similar magnetic characteristic. This suggests that the fields of these stars are not being contemporaneously generated but are instead a remnant from a previous phase, or event, that occurred earlier during stellar evolution or even formation (e.g. Wade et al. and Grunhut et al. these proceedings). Moreover, since such large-scale magnetism is not a ubiquitous feature for stars with radiative envelopes, it cannot explain that most massive stars have X-ray emission.

Second, even if shallow sub-surface convection layers associated with opacity peaks of iron (Cantiello et al. 2009) could excite stochastic pulsations and perhaps provide mechanical heating at the surface, massive stars still would not have hot coronae. Indeed, their radiatively-driven winds are orders of magnitude denser than solar-type stars. As the wind densities are not low enough for a thermal runaway to occur, their winds stay cool, close to the surface effective temperature (Drew 1989).

Therefore, even OB stars that possess large-scale magnetic fields do not emit X-rays through the same coronal process as low-mass stars. Instead, X-ray production for massive stars is generally related to their strong winds, more specifically from wind material that has been shock-heated by various mechanisms, as reviewed in the following section.

\section{X-ray emission processes for OB stars}

Most massive OB stars emit relatively soft, stable X-rays $(0.5 \mathrm{keV})$ when compared with low-mass stars (a few keV). They are very bright, $L_{\mathrm{X}} \sim 10^{31}-10^{33} \mathrm{erg} \mathrm{s}^{-1}$, but this X-ray emission only represents $\sim 10^{-7}$ of their bolometric luminosity (Nazé et al. 2011).

This reflects the fact that the most universal shock mechanism, which heats up a few percent of the wind material, is caused by velocity variations in the supersonic wind 
outflow, intrinsic to the unstable nature of the radiative line-driving (Owocki, Castor \& Rybicki 1988, Feldmeier, Puls \& Pauldrach 1997). The low shock-jump velocities of these embedded wind shocks (EWS) hence create soft emission that is distributed throughout the whole wind in such a way to produce temporally nearly steady X-ray flux and Doppler-broadened ( $>1500 \mathrm{~km} / \mathrm{s}$ ) emission lines (e.g. Nazé et al. 2013, Cohen et al. 2010).

Massive star binaries with collision between their mutual winds at near their terminal speed $(\sim 2000 \mathrm{~km} / \mathrm{s})$ also can in some cases lead to strong emission $\left(L_{\mathrm{X}} \sim 10^{31}-10^{34}\right)$ of much harder X-rays (up to $5-10 \mathrm{keV}$ ) that will often display drastic variability tied to the orbital period (e.g. Corcoran et al. 2010, de Becker et al. 2006).

Finally, for stars hosting magnetic fields at their surface, X-rays can be produced through the Magnetically Confined Wind Shock model (MCWS; Babel \& Montmerle 1997, ud-Doula \& Owocki 2002). In this paradigm, the star's radiation-driven wind is channeled by a large-scale dipole magnetic field such that material is forced to flow along the field lines and collide near the tops of closed loops, producing a shock-heated volume of plasma. Although such shocks are nearly head-on, the magnetic field can only confine the wind up to an Alfvén radius $R_{\mathrm{A}} \approx\left(B_{\text {eq }} R_{\star}\right)^{1 / 2} /\left(\dot{M} v_{\infty}\right)^{1 / 4}$ and the winds might not have been fully accelerated before reaching the top of the highest loop. Therefore, the $\mathrm{X}$-rays are expected to be softer than for binaries, but still more luminous and energetic than single non-magnetic stars. Furthermore, if the magnetic axis is tilted with respect to the rotational axis, it is possible to observe modulation of the X-ray emission over the rotational period, such as seen for the magnetic O-type star $\theta^{1}$ Ori C (Gagné et al. 2005).

\section{X-rays from magnetic OB stars}

X-ray observations are valuable to our understanding of magnetic massive stars, as they trace the hot gas and provide constraints on the kinematics and shocks in these magnetospheres.

For example, Fig. 1 compares the X-ray high-resolution spectra of the non-magnetic O-type star $\zeta$ Pup, the magnetic O-type star $\theta^{1}$ Ori $\mathrm{C}$, and the coronal emission from the G-type star Capella, obtained by the High Energy Transmission Gratings (HETG) aboard the Chandra X-ray Observatory. The emission lines of $\zeta$ Pup are significantly Doppler-broaden, consistent with the expectation of EWS. As can been seen from a comparison with the unresolved coronal lines of Capella, the emission lines of $\theta^{1}$ Ori C are much narrower, although still resolved, indicative of the low, but non-zero post-shock velocities expected for MCWS.

The X-ray emission from the population of magnetic OB stars as a whole can also guide the models of magnetospheres by examining trends in X-ray luminosity (and eventually $\mathrm{X}$-ray temperature) as a function of stellar/magnetic parameters, such as those predicted by the parameter study presented by Owocki et al. (these proceedings, also ud-Doula et al. in prep). Such studies could help explain the wide-ranging X-ray properties of magnetic stars that do not all conform at first glance to the simple MCWS paradigm (e.g. Petit 2011, Oskinova et al. 2011, Nazé et al. 2010).

For example, Fig. 2 presents all available X-ray efficiencies $\log \left(L_{\mathrm{X}} / L_{\mathrm{bol}}\right)$ of hot magnetic OB stars, from the compilation by Petit et al. (2013). For stars without centrifugal support of the magnetically trapped material (dynamical magnetosphere), a trend of increased X-ray luminosity with mass-loss rate (which is a function of stellar bolometric luminosity) is expected, as illustrated by the left panel of Fig. 2. For stars with fast enough rotation such that the Kepler radius $\left(R_{\mathrm{K}}\right.$; material forced in co-rotation above this radius is centrifugally supported against gravity) is closer to the surface than the Alfvén radius (right panel of Fig. 2), the same trend with luminosity is observed. But 

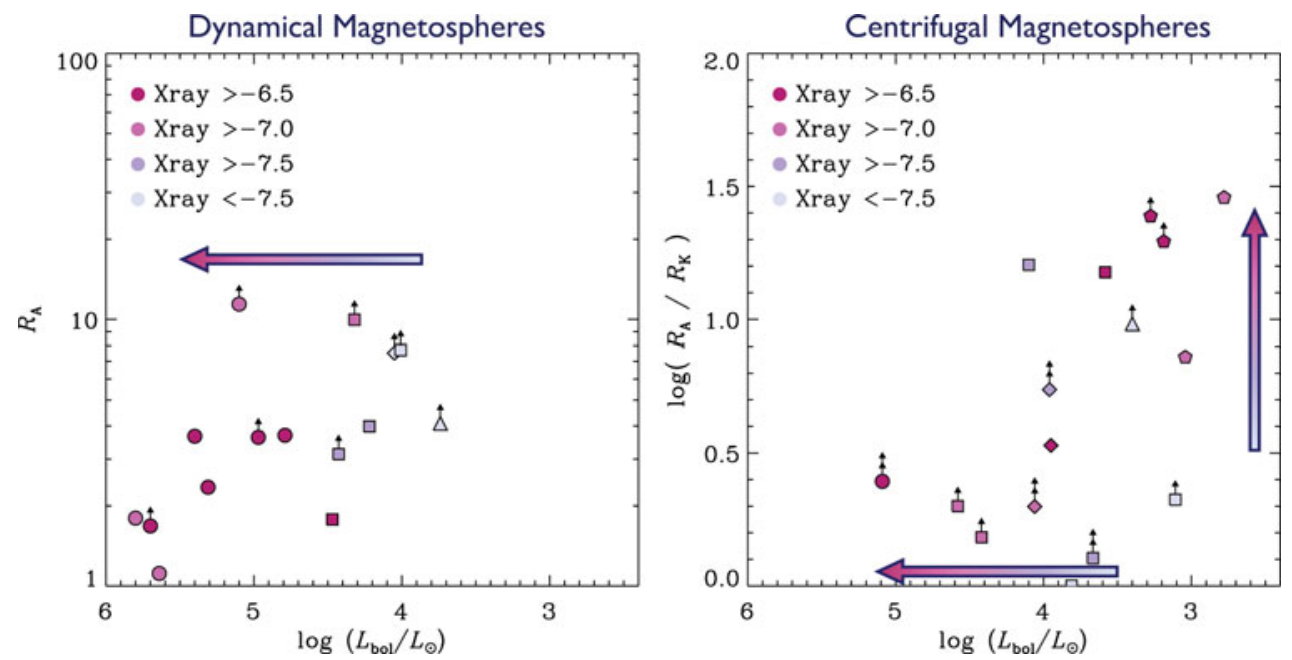

Figure 2. Left: X-ray efficiency $\left(\log \left(L_{\mathrm{X}} / L_{\mathrm{bol}}\right)\right.$; colour scale) of hot magnetic OB stars with dynamical magnetospheres as a function of their position in a diagram of Alfvén radius $\left(R_{\mathrm{A}}\right)$ versus luminosity (as a proxy for mass-loss rate). Right: X-ray efficiency of stars with centrifugal magnetospheres as a function of the position in a diagram of the ratio of the Alfvén radius to the Kepler co-rotation radius $R_{\mathrm{K}}$ (as a proxy for the size of the supported magnetospheric region) versus the bolometric luminosity. The expected qualitative trends are illustrated with arrows.

there also seems to be a trend of enhanced X-ray luminosity with the size of the centrifugally supported region (vertical axis), which could be explained by an enhancement of the shock-jump velocities from centrifugal acceleration.

\section{References}

Babel, J. \& Montmerle, T. 1997 A\&A, 323, 121

Berghoefer, T. W., Schmitt, J. H. M. M., Danner, R., \& Cassinelli, J. P. 1997, A\&GA, 322, 167

Cantiello, M. et al. 2009, A\&̊A, 499, 279

Cohen, D. H., Leutenegger, M. A., Wollman, E. E., Zsargó, J., Hillier, D. J., Townsend, R. H. D., \& Owocki, S. P. 2010, MNRAS, 405, 2391

Corcoran, M. F., Hamaguchi, K., Pittard, J. M., Russell, C. M. P., Owocki, S. P., Parkin, E. R., \& Okazaki, A. 2010, ApJ, 725, 1528

De Becker et al. 2006, MNRAS, 371, 1280

Drew, J. E. 1989 ApJS, 71, 267

Feldmeier, A., Puls, J., \& Pauldrach, A. W. A. 1997, A\&A, 322, 878

Gagné, M., et al. 2005, ApJ, 628, 986

Nazé, Y., Oskinova, L. M., \& Gosset, E. 2013, ApJ, 763, 143

Nazé, Y. et al. 2011, ApJS 194, 7

Nazé, Y., ud-Doula, A., Spano, M., Rauw, G., De Becker, M., Walborn, N. R. 2010 520, A59

Oskinova, L. M., Todt, H., Ignace, R., Brown, J. C., Cassinelli, J. P., Hamann, W.-R. 2011 $M N R A S, 416,1456$

Owocki, S. P., Castor, J. I., \& Rybicki, G. B. 1988, ApJ, 335, 914

Petit, V. et al. 2013, MNRAS, 429, 398

Petit, V. 2011 Ph.D. Thesis, Université Laval (Canada)

Power, J. 2007, Ph.D. Thesis, Queen's University (Canada)

ud-Doula, A. \& Owocki, S. P. 2002, ApJ, 576, 413

Wade, G. A. et al. 2011 in IAU 272, 118

Wright, N. J., Drake, J. J., Mamajek, E. E., \& Henry, G. W. 2011, ApJ, 743, 48 